\title{
Asymptotically Safe gravitational collapse: Kuroda-Papapetrou RG-improved model
}

\author{
A. Bonanno \\ INAF, Osservatorio Astrofisico di Catania, via S. Sofia 78, I-95123 Catania, Italy \\ INFN, Sezione di Catania, via S. Sofia 64, I-95123, Catania, Italy \\ E-mail: alfio.bonanno@oact.inaf.it

\section{B. Koch} \\ Instituto de Física, Pontificia Universidad Católica de Chile, Av. Vicuna Mackenna \\ 4860, Santiago, Chile \\ E-mail: bkoch@fis.puc.cl
}

\section{A. Platania*}

Università di Catania, via S. Sofia 63, I-95123 Catania, Italy

INAF, Osservatorio Astrofisico di Catania, via S. Sofia 78, I-95123 Catania, Italy

INFN, Sezione di Catania, via S. Sofia 64, I-95123, Catania, Italy

Institute for Mathematics, Astrophysics and Particle Physics (IMAPP), Radboud

University Nijmegen, Heyendaalseweg 135, 6525 AJ Nijmegen, The Netherlands

E-mail: alessia.platania@oact.inaf.it

\begin{abstract}
According to Wheeler, the gravitational collapse is the "greatest crisis in Physics of all time". In this work the structure of the singularity produced by a collapse of a nullfluid will be discussed in the framework of Asymptotically Safe (AS) Gravity. Under the assumption of spherical symmetry, it will be shown that the generic singularity arising in the collapse of a null-dust is spacelike and "whimper", following the Tipler classification, allowing in principle an extension of the spacetime across the singularity.
\end{abstract}

Corfu Summer Institute 2016 "School and Workshops on Elementary Particle Physics and Gravity"

31 August - 23 September, 2016

Corfu, Greece

${ }^{*}$ Speaker. 


\section{Introduction}

According to the Cosmic Censorship Conjecture (CCC) all singularities in physically realistic spacetimes are hidden inside an event horizon [1]. CCC thus implies that no signal from the singularity should be able to reach null infinity. The original argument of Penrose did not specify or constraint the type of singularities that are the subject to CCC, and it was in fact soon realized that several counterexamples to CCC can be easily constructed depending on the details of the collapse. Leaving aside the possibility of defining the precise set of initial conditions and matter field which do not lead to the presence of naked singularities, one can argue that a consistent theory of quantum gravity can provide a different solution to the problem of singularities in classical General Relativity.

In recent years it has been realized that a promising approach to quantum gravity is the Asymptotic Safety scenario. The idea, as first proposed by Weinberg [2], is that General Relativity can have a consistent and predictive ultraviolet completion around an UV-attractive Non-Gaussian Fixed Point (NGFP) [3] which renders the theory finite (for a recent review see [4]).

The aim of this work is to study the effects of quantum gravity on the nature of the singularity arising from the gravitational collapse of a null fluid, complementing the analysis in [5]. The idea is to perform a RG-improvement of the well known Vaidya-KurodaPapapetrou (VKP) model [6-9] and follow the analysis in [10] to study the possibility to extend the spacetime beyond the singular point. In particular we found that the usual singularity arising from the gravitational collapse is replaced by an "integrable" singularity [11], and that the Cosmic Censorship violation cannot be avoided even including quantum gravity effects.

This work is organized as follows. In section 2 we shall begin by introducing the classical description of the gravitational collapse within the VKP model. The RG-improvement procedure in the context of Asymptotically Safe gravity and the construction of the quantum corrected metric are presented in section 3. In section 4 we discuss our findings on the singularity nature in the RG-improved Vaidya spacetime, while section 5 is a short summary of our results and their possible consequences and extensions.

\section{Vaidya-Kuroda-Papapetrou spacetimes}

As it is well known, the Schwarzschild vacuum solution of Einstein equations describes the spacetime structure around a spherical massive object such as a star. This solution has an unphysical singularity at the Schwarzschild radius $r_{s}=2 G_{0} m$, that is removable with a change of coordinates. In this respect it is useful to introduce the ingoing EddingtonFinkelstein coordinates, in which the time coordinate is replaced by the advanced time parameter $v=t+r^{*}$, with

$$
r^{*} \equiv r+r_{s} \ln \left|\frac{r}{r_{s}}-1\right|
$$

In particular, in order to describe dynamical spacetimes, e.g. the gravitational collapse, it is reasonable to assume that the mass of the object is instead a mass function $m(v)$ and 
thus the metric assumes the form

$$
d s^{2}=-f(r, v) d v^{2}+2 d v d r+r^{2} d \Omega^{2}
$$

with the lapse function $f(r, v)$ given by

$$
f(r, v)=1-\frac{2 G_{0} m(v)}{r}
$$

This solution, known as Vaidya spacetime [6], describes the spacetime around a spherically symmetric object with variable mass $m(v)$. The metric $(2.2)$ is an exact solution of the Einstein field equations in presence of a Type II fluid, and thus it is perfectly suited to describe the gravitational collapse of a massive star. In particular, the collapse can be modeled as the implosion of a series of radiation shells, represented by the world lines $v=$ cost. In the Vaidya-Kuroda-Papapetrou (VKP) model $[8,9]$ the mass function is parametrized as follows

$$
m(v)= \begin{cases}0 & v<0 \\ \lambda v & 0 \leq v<\bar{v} \\ \bar{m} & v \geq \bar{v}\end{cases}
$$

For $v<0$ the spacetime is flat and empty (Minkowski spacetime), then the collapse starts and the radiation shells are injected and focused towards $r=0$, causing the mass to grow as $m(v)=\lambda v$. As the null dust collapses into $r=0$, the VKP spacetime develops a persistent central singularity, and whether this singularity is covered or not by an event horizon depends on the collapse dynamics. When the process ends, in $v=\bar{v}$, the metric reduces to the Schwarzschild static solution, with final mass $\bar{m}$.

The outcome of the collapse can be studied by solving the geodesic equation for null outgoing light rays, as it gives information on the causal structure of the spacetime. The general solution of the geodesic equation can be written as [12-14]

$$
\frac{\left|r(v)-\mu_{-} v\right|^{\mu_{-}}}{\left|r(v)-\mu_{+} v\right|^{\mu_{+}}}=\tilde{C}
$$

where $\tilde{C}$ is a complex constant and

$$
\mu_{ \pm}=\frac{1 \pm \sqrt{1-16 \lambda G_{0}}}{4}
$$

This solution is characterized by a critical value of the radiation rate, $\lambda_{c} \equiv 1 / 16 G_{0}$, below which it is always possible to causally connect the central singularity $r=0$ with an external observer. Therefore if the initial conditions of the physical system entails $\lambda \leq \lambda_{c}$, the gravitational collapse will result in a naked singularity and thus the Cosmic Censor Conjecture would be violated in its weak formulation. 


\section{RG-improved VKP spacetimes}

In this section we briefly review the main results obtained in [5]. The idea is to study the leading quantum effects produced by a running Newton's constant [15], by using the "RG-improvement" procedure. The non-perturbative Renormalization Group techniques allow in fact to compute the evolution of the effective average action $\Gamma_{k}\left[g_{\mu \nu}\right]$ via the exact functional equation [16]

$$
k \partial_{k} \Gamma_{k}=\frac{1}{2} \mathrm{~S} \operatorname{Tr} \frac{\partial_{k} \mathcal{R}_{k}}{\Gamma_{k}^{(2)}+\mathcal{R}_{k}}
$$

This equation describes the flow in energy of $\Gamma_{k}$ through the theory space, and allows to connect continuously the high energy regime, described by the classical action $S=\Gamma_{\infty}$, with the infrared limit $k \rightarrow 0$, which is described by the usual effective action $\Gamma_{0}$. Once an ansatz for the gravitational part of the action is chosen, it is possible to project the flow on the correspondent subspace and compute the flow equation for each coupling constant. Under the Einstein-Hilbert approximation it was found in [15] that the scaling of the Newton's constant is approximately given by

$$
G(k)=\frac{G_{0}}{1+\omega G_{0} k^{2}}
$$

where $\omega=1 / g_{*}$, being $g_{*}$ the value of the dimensionless Newton's constant at the ultraviolet NGFP [3], and $k$ is the infrared cutoff scale appearing in eq. (3.1). As it will be important for our discussion, we want to remark that the dimensionful Newton's coupling (3.2) vanishes in the ultraviolet limit $k \rightarrow \infty$.

In order to take into account the main quantum contributions in the description of the gravitational collapse, the key idea is to use the classical VKP solution (2.2) and perform the RG-improvement via the replacement

$$
f(r, v) \longrightarrow f_{\mathrm{I}}(r, v)=1-\frac{2 m(v)}{r} \frac{G_{0}}{1+\omega G_{0}[k(r)]^{2}}
$$

It is important to notice that in order to have a consistent description of the Vaidya spacetime modified by a running Newton's constant, the infrared cutoff must be associated with a function $k(r)$ relating the mathematical scaling of the Newton's constant with the actual collapse dynamics. In the case at hand the only reasonable energy scale involved, characterizing the collapsing fluid and the singularity formation, is the radiation energy density $\rho(r)$. With a simple dimensional analysis argument one can argue that $k(r)=$ $\xi \sqrt[4]{\rho(r)}$, where $\xi$ is a positive constant and $\rho=\dot{m}(v) / 4 \pi r^{2}$. The improved lapse function thus reads

$$
f_{\mathrm{I}}(r, v)=1-\frac{2 m(v) G_{0}}{r+\alpha \sqrt{\lambda}} \quad \alpha=\frac{\xi^{2} G_{0}}{\sqrt{4 \pi} g_{*}}
$$

where $m(v)$ is the mass function introduced in eq. (2.4). The metric defined by the RGimproved lapse function (3.4) is part of a larger class of metrics, known as generalized 
Vaidya spacetimes, in which $f(r, v)$ has the following form

$$
f(r, v)=1-\frac{2 M(r, v)}{r}
$$

The generalized Vaidya spacetimes are thus characterized by a generalized mass function $M(r, v)$ that depends on both the advanced time and the radial coordinate. This family of metrics, first introduced by Wang and $\mathrm{Wu}$ [17], corresponds to an exact solution of Einstein field equations associated with a mixture of Type-I and Type-II fluids, whose stress-energy tensor is

$$
T_{\mu \nu}=\underbrace{\rho l_{\mu} l_{\nu}}_{\text {Type II }}+\underbrace{(\sigma+p)\left(l_{\mu} n_{\nu}+l_{\nu} n_{\nu}\right)+p g_{\mu \nu}}_{\text {Type I }}
$$

with $n_{\mu} l^{\mu}=-1, l_{\mu} l^{\nu}=0$ and

$$
\begin{gathered}
\rho(r, v)=\frac{1}{4 \pi G_{0} r^{2}} \frac{\partial M(r, v)}{\partial v} \\
\sigma(r, v)=\frac{1}{4 \pi G_{0} r^{2}} \frac{\partial M(r, v)}{\partial r}, \quad p(r, v)=-\frac{1}{8 \pi G_{0} r} \frac{\partial^{2} M(r, v)}{\partial r^{2}}
\end{gathered}
$$

In the classical VKP model $M(r, v)=G_{0} m(v)$, so that the stress-energy tensor (3.6) reduces to the pure Type II one, while the RG-improved counterpart has $M(r, v)=G(r) m(v)$, that corresponds to a non-trivial fluids mixture [5].

The structure of the spacetime, the singularity formation and the validity of the Cosmic Censorship Hypothesis can be studied, as in the classical case, by solving the geodesic equation for an outgoing null observer. The RG-improved geodesic equation is

$$
\dot{r}(v)=\frac{1}{2}\left(1-\frac{2 m(v) G_{0}}{r(v)+\alpha \sqrt{\lambda}}\right)
$$

It differs from the classical equation only for a shift in the radial coordinate $r(v)$. As it was found in [5] this shift leads to an increase of the critical value $\lambda_{c}$, favoring the formation of naked singularities. Furthermore, as it shown in Fig. 1, the event horizon forms well after the formation of the singularity, thus allowing the singularity to be naked and persistent along the advanced time $v$ direction.

\section{Singularity and integrability of the RG-improved VKP model}

In the singularity theorems nothing is specified on the "nature" of the singularities. The real problem caused by spacetime singularities is not the divergence of physical quantities, but instead the impossibility to know the future evolution of geodesics beyond the singularity (a radially infalling observer will suddenly disappear from the spacetime once the singularity is reached). It is this feature that breaks the physical predictability, and thus the validity of General Relativity as a classical deterministic theory.

As discussed in $[10,18]$, the physical relevance of a spacetime singularity is determined by its strength. By following the Tipler classification [11] a singularity is called "strong" 


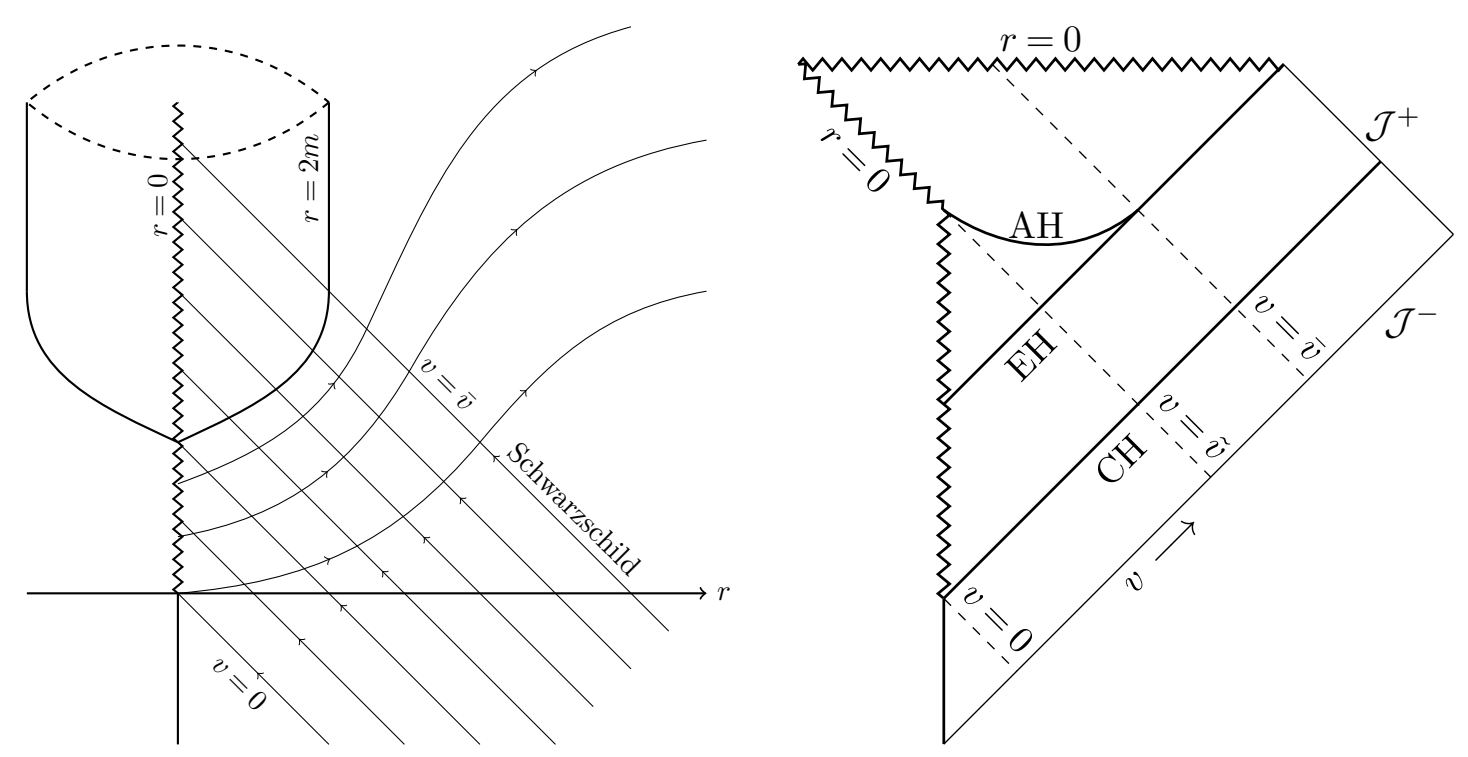

Figure 1: Global structure of the spacetime for $\lambda \leq \lambda_{c}$.

if any object sent into this spacetime point is destroyed by the (infinite) gravitational tidal forces. A strong curvature singularity is the only physical relevant singularity: if the singularity is instead "integrable" one can extend the spacetime beyond it, rendering every non-spacelike geodesic well defined for all values of the affine parameter.

A way to determine the appearance of naked singularities and mathematically characterize their strength was developed in [10]. In the generalized Vaidya spacetime the lapse function $f(r, v)$ is defined by eq. (3.5), and the corresponding geodesic equation for null rays can be written in the form of a dynamical system in terms of an affine parameter $t$

$$
\left\{\begin{array}{l}
\frac{\mathrm{d} v(t)}{\mathrm{d} t}=2 r \\
\frac{\mathrm{d} r(t)}{\mathrm{d} t}=r-2 M(r, v)
\end{array}\right.
$$

The fixed point solution of the system is $r=0$, with the additional constraint that the generalized mass function vanishes on the hypersurface $r=0, M(0, v)=0$. This set of solutions define the "points" where the spacetime is singular. The behavior of the trajectories in the vicinity of the singularity is determined by the eigenvalues of the stability matrix (Jacobian) evaluated at the fixed point (FP)

$$
\chi_{ \pm}=\frac{1}{2}\left(\operatorname{Tr} J \pm \sqrt{(\operatorname{Tr} J)^{2}-4 \operatorname{det} J}\right)
$$

with $\operatorname{Tr} J=1-2\left(\partial_{r} M\right)_{\mathrm{FP}}$ and $\operatorname{det} J=4\left(\partial_{v} M\right)_{\mathrm{FP}}$, and the correspondent characteristic lines

$$
r_{ \pm}(v)=r_{\mathrm{FP}}+\frac{\chi_{ \pm}}{2}\left(v-v_{\mathrm{FP}}\right)
$$

which define how radial null geodesics approach the fixed point. The central singularity is locally naked if there exists at least one non-spacelike geodesic starting from the hypersur- 
face $r=0$ with a well defined tangent, and reaching the future null infinity. In terms of the dynamical system analysis this condition is verified if the fixed point FP is a repulsive node. Furthermore, according to [10], the singularity strength can be characterized by the parameter

$$
S=\frac{X_{\mathrm{FP}}^{2}}{2}\left(\partial_{v} M\right)_{\mathrm{FP}}, \quad X_{\mathrm{FP}} \equiv \lim _{(r, v) \rightarrow \mathrm{FP}} \frac{v(r)}{r}
$$

The fixed point solution corresponds to a strong curvature singularity if $S$ is positive, while for $S \leq 0$ the singularity is integrable.

In the RG-improved VKP model the generalized mass function is given by

$$
M(r, v)=m(v) G(r)
$$

where the running Newton's constant $G(r)$ is obtained from eq. (3.2) by replacing $k$ with the infrared cutoff function

$$
k(r)=\xi \sqrt[4]{\frac{\dot{m}(v)}{4 \pi r^{2}}}
$$

Since the condition $M(0, v)=0$ is verified for all values of the advanced time $v$, the fixed point solution of the geodesic equation is the line $r=0$. This circumstance is realized because the determinant of the stability matrix is zero, and this latter condition is due to the anti-screening behavior of the running Newton's constant in the high energy regime

$$
\operatorname{det} J \propto\left(\partial_{v} M\right)_{\mathrm{FP}} \propto \lim _{r \rightarrow 0} G(r)=0
$$

Furthermore, this feature implies a vanishing value of the strength parameter

$$
S \propto\left(\partial_{v} M\right)_{\mathrm{FP}}=0
$$

Thus the effect of a running Newton's constant vanishing in the ultraviolet limit is to turn the strong curvature singularity of the classical VKP model into a line of integrable singularities.

The fixed points line is characterized by one marginal direction $r=0$, along which the fixed points are located, and by a family of non-marginal characteristic lines whose slope depends on the precise location of the fixed point $\left(0, v_{0}\right)$

$$
v=v_{0}+\frac{2 r}{\chi_{+}\left(v_{0}\right)}, \quad \chi_{+}\left(v_{0}\right) \equiv \operatorname{Tr} J=1-\frac{2 \lambda v_{0} G_{0}}{\alpha \sqrt{\lambda}}
$$

For a given fixed point $P \equiv\left(0, v_{0}\right)$, the curve in eq. (4.9) describes the tangent line to the trajectory ending in $P$. Depending on $v_{0}$ the slope of such lines can be either positive or negative, and the value $\bar{v}_{0}$ at which the slope inverts its sign corresponds to the condition

$$
r_{\mathrm{AH}}\left(\bar{v}_{0}\right)=0
$$

Moreover, since $\chi_{+}\left(v_{0}\right) \equiv \operatorname{Tr} J$, if the slope of (4.9) in $P$ is positive (negative) the fixed point $P \equiv\left(0, v_{0}\right)$ is repulsive (attractive) along the correspondent non-marginal character- 


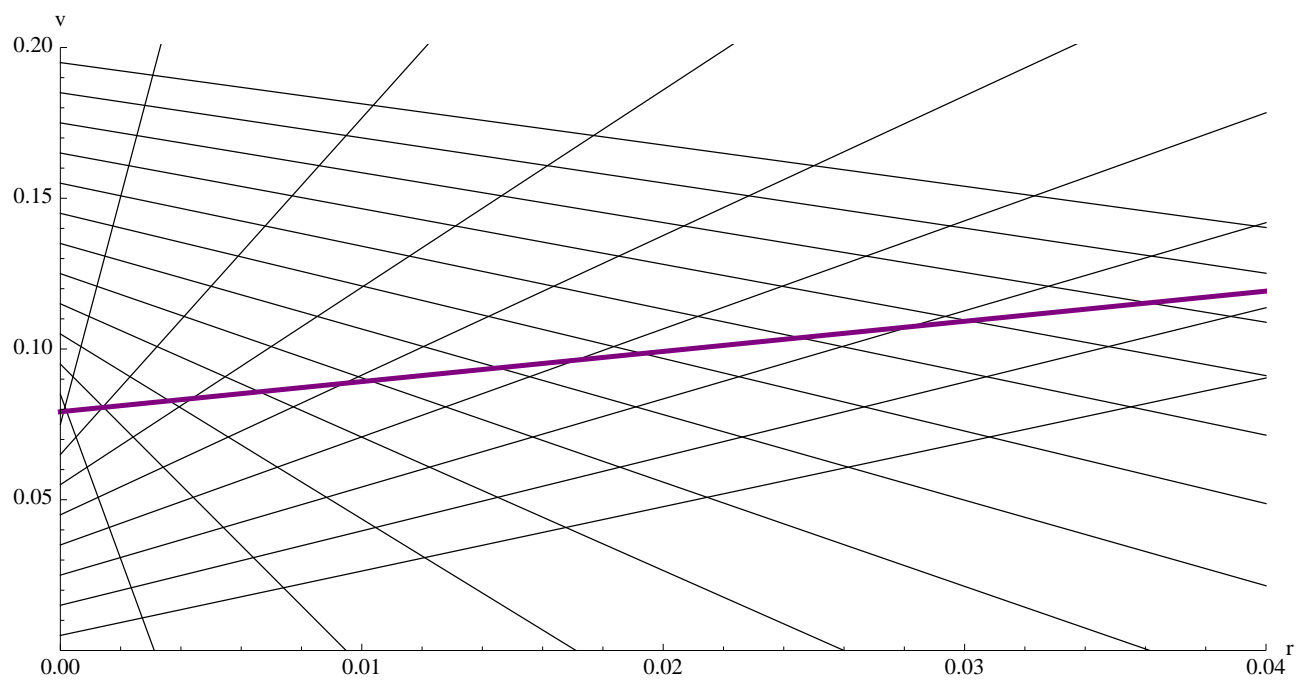

Figure 2: Characteristic lines controlling the behavior of the system near the singular line, eq. (4.9). The trajectories that start above (under) the apparent horizon (purple line) have negative (positive) slope. The qualitative behavior of these characteristic directions does not depend on $\lambda$.

istic direction. The configuration of the characteristic lines is shown in fig. 2. Clearly the characteristic curves cannot cross each other, and the appearance of intersections is an artifact of the description used, which is based on the linearization of the geodesic equation around $r=0$. Far from the fixed points line the non-linearity effects of the original dynamical system must fold and join the characteristic lines so that different solutions never intersect each other. It is interesting to notice that in this analysis the qualitative behavior of the trajectories does not depend on the radiation rate $\lambda$. Every fixed point $P$ along the marginal direction $r=0$ has a zero eigenvalue, $\chi_{-}=0$, and therefore this continuous set of fixed points forms a line of improper nodes for all values of $\lambda$. It follows that, according to the definition, the singularity in the RG-improved model is not locally naked. This statement seems to contradict the results in [5], in which it was found that there exists a critical value $\lambda_{c}$ below which the singularity is naked, whose value is greater than the classical one. The apparent mismatch is related to the fact that the analytical solutions found in [5] are obtained by solving the full geodesic equation, while the study of the singularity with the approach of [10] is performed by linearizing the system around $r=0$.

The critical value of the radiation rate is restored once the full RG-improved geodesic equation is considered. In this case the family of characteristic curves in eq. (4.9) becomes a continuum set of heteroclinic orbits between couple of attractive-repulsive fixed points, as it is shown in fig. 3 for $\lambda>\lambda_{c}$. Moreover, the study of the full geodesic equation allows to interpret the fixed points line and the correspondent $v_{0}$-dependent characteristic curves as result of a spiral node located in the unphysical part of the diagram $(r<0)$. In fact, the RG-improved geodesic equation can be obtained from the classical one by shifting the radial coordinate $r(v) \rightarrow r(v)+\alpha \sqrt{\lambda}$, and since the classical VKP model presents a singularity in $P \equiv(0,0)$, which is a spiral node for $\lambda>\lambda_{c}$, this node has the effect of producing a line of fixed points $(r=0)$ in the RG-improved (shifted) system. 

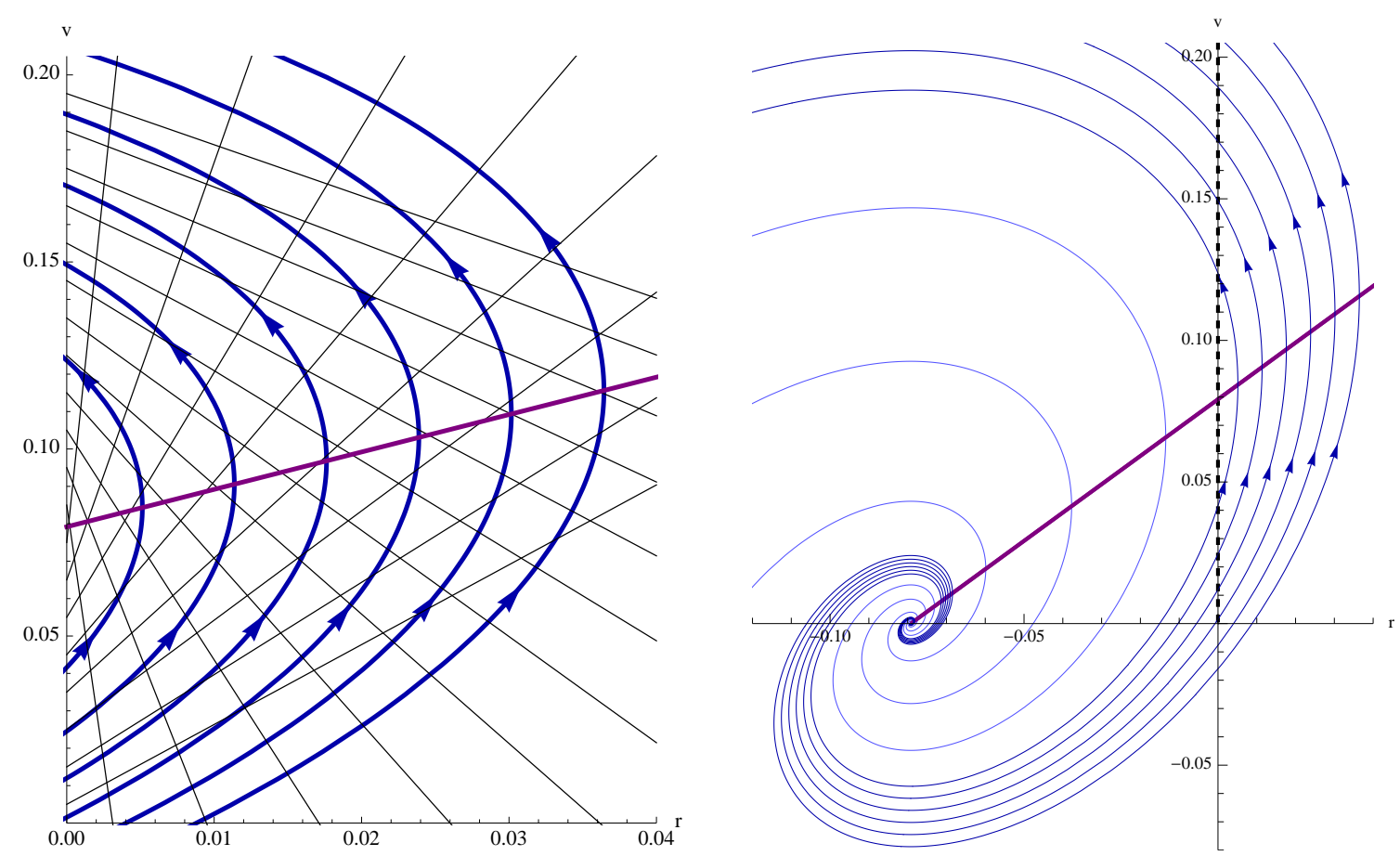

Figure 3: Solutions of the full RG-improved geodesic equation for $\lambda>\lambda_{c}$. Left panel: the nonlinearity effects fold and join the characteristic lines (black lines), resulting in a set of characteristic curves (blue lines) linking couple of attractive-repulsive fixed points. Right panel: the line of fixed points and the correspondent characteristic curves can also be understood in terms of the "shifted classical solution", as they are generated by the shifted spiral node characterizing the classical VKP model for $\lambda>\lambda_{c}$.

\section{Conclusions}

In this communication an RG-improved Vaidya model of gravitational collapse has been proposed. In particular it has been shown that the effect of a running Newton's constant, according to the AS scenario in Quantum Gravity, is to dramatically alter the strength of the singularity at $r=0$ of a non-rotating model, resulting in "whimper" type of singularity. It would be important to extend our findings including the presence of angular momentum and discuss possible astrophysical implications of our model. We hope to address these issues in a future work.

\section{References}

[1] R. Penrose. In: Rivista del Nuovo Cimento 1 (1969), p. 252.

[2] S. Weinberg. "Critical Phenomena for Field Theorists." In: Erice Subnuclear Physics (1976).

[3] M. Reuter. "Nonperturbative evolution equation for quantum gravity." In: Phys. Rev. D 57 (Jan. 1998), pp. 971-985. DOI: 10.1103/PhysRevD . 57 . 971. eprint: hep-th/ 9605030. 
[4] M. Reuter and F. Saueressig. "Quantum Einstein gravity." In: New Journal of Physics 14.5, 055022 (May 2012), p. 055022. DOI: 10.1088/1367-2630/14/5/055022. arXiv: 1202.2274 [hep-th].

[5] A. Bonanno, B. Koch, and A. Platania. "Cosmic Censorship in Quantum Einstein Gravity." In: ArXiv e-prints (Oct. 2016). arXiv: 1610.05299 [gr-qc].

[6] P. C. Vaidya. "Nonstatic Solutions of Einstein's Field Equations for Spheres of Fluids Radiating Energy." In: Physical Review 83 (July 1951), pp. 10-17. DOI: 10.1103/ PhysRev. 83.10.

[7] P. C. Vaidya. "An Analytical Solution for Gravitational Collapse with Radiation." In: ApJ 144 (June 1966), p. 943. DOI: 10.1086/148692.

[8] Y. Kuroda. "Naked Singularities in the Vaidya Spacetime." In: Progress of Theoretical Physics 72 (July 1984), pp. 63-72. DOI: 10.1143/PTP.72.63.

[9] A. Papapetrou. "Formation of a singularity and causality." In: A Random Walk in Relativity and Cosmology. Ed. by M. Dadhich et al. 1985, pp. 184-191.

[10] M. D. Mkenyeleye, R. Goswami, and S. D. Maharaj. "Gravitational collapse of generalized Vaidya spacetime." In: Phys. Rev. D 90.6, 064034 (Sept. 2014), p. 064034. DOI: $10.1103 /$ PhysRevD. 90.064034.

[11] F. J. Tipler. "On the nature of singularities in general relativity." In: Phys. Rev. D 15 (Feb. 1977), pp. 942-945. DOI: 10.1103/PhysRevD.15.942.

[12] W. Israel. "The formation of black holes in nonspherical collapse and cosmic censorship." In: Canadian Journal of Physics 64 (Feb. 1986), pp. 120-127. DOI: 10.1139/ p86-018.

[13] I. H. Dwivedi and P. S. Joshi. "On the nature of naked singularities in Vaidya spacetimes." In: Classical and Quantum Gravity 6 (Nov. 1989), pp. 1599-1606. DOI: 10.1088/0264-9381/6/11/013.

[14] I. H. Dwivedi and P. S. Joshi. "On the nature of naked singularities in Vaidya spacetimes: II." In: Classical and Quantum Gravity 8.7 (1991), p. 1339. URL: http: / / stacks. iop.org/0264-9381/8/i=7/a=010.

[15] A. Bonanno and M. Reuter. "Renormalization group improved black hole spacetimes." In: Phys. Rev. D 62.4, 043008 (Aug. 2000), p. 043008. DOI: 10 . 1103 / PhysRevD.62.043008. eprint: hep-th/0002196.

[16] C. Wetterich. "Exact evolution equation for the effective potential." In: Physics Letters B 301 (Feb. 1993), pp. 90-94. DOI: 10.1016/0370-2693(93) 90726-X.

[17] A. Wang and Y. Wu. "Generalized Vaidya Solutions." In: General Relativity and Gravitation 31 (Jan. 1999), pp. 107-114. DOI: 10.1023/A : 1018819521971. eprint: gr-qc/9803038.

[18] P. S. Joshi. Gravitational Collapse and Spacetime Singularities. Sept. 2012. 